\title{
ANTIBIOTIC-ELUTING RESORBABLE BONE-VOID FILLER EVALUATED IN A LARGE ANIMAL INFECTION PREVENTION MODEL
}

\author{
Z. Ferrell ${ }^{1}$, D.W. Grainger ${ }^{1,2}$ and K.D. Sinclair ${ }^{1,3 *}$ \\ ${ }^{1}$ Department of Biomedical Engineering, University of Utah, Salt Lake City, UT 84112, USA \\ ${ }^{2}$ Department of Pharmaceutics and Pharmaceutical Chemistry, University of Utah, Salt Lake City, \\ UT 84112, USA \\ ${ }^{3}$ Elute, Inc., 417 Wakara Way, Salt Lake City, UT 84108, USA
}

\begin{abstract}
Periprosthetic infection in total knee arthroplasty is a difficult-to-treat complication. Current implant revision procedures use non-degradable, antibiotic-loaded bone cement for local antimicrobial delivery. As a permanent foreign body, antibiotic-loaded bone cement is susceptible to bacterial colonisation after antibiotic release. In this first step, of a multi-study approach, an infection prevention model assessed a resorbable, antibiotic-eluting bone-void filler for preventing infection in a large animal model. Four groups of sheep were utilised to monitor antibiotic-eluting bone-void filler-induced osteoconductivity, infection prevention, and implant resorption. Explanted bone and surrounding tissues were evaluated using quantitative microbiology, backscattered electron microscopy, bone mineral apposition, and Sanderson's staining at the 12-week endpoint. Control groups received commercially available bone-void filler, implanted into a surgically created defect on the right medial femoral condyle. Experimental groups received six antibiotic-eluting bone-void filler devices placed into identically sized defects. One control and one experimental group tested osteoconductivity. An additional control and experimental group were each inoculated with $5 \times 10^{5}$ colony forming units $/ \mathrm{mL}$ Staphylococcus aureus during implant placement for bactericidal effects. Osteoconductivity was confirmed for both antibiotic-eluting bone-void filler and commercially available bone-void filler. The experimental group inoculated with S. aureus showed no detectable bacteria at the study's 12-week endpoint, while infection controls required euthanasia 6-11 d post-inoculation due to infection. This large animal study validated this antibiotic-eluting bone-void filler as osteoconductive, in situ degradable, and bactericidal. All groups, except the infection control, exhibited bone formation comparable to commercial filler ProOsteon ${ }^{\circledR 500 R}$.
\end{abstract}

Keywords: Animal model, periprosthetic infection, controlled antimicrobial release, bone void filler, tobramycin.

*Address for Correspondence: K.D. Sinclair, Elute, Inc., 417 Wakara Way, Salt Lake City, UT 84108, USA Email: sinclairkristof@gmail.com

Copyright policy: This article is distributed in accordance with Creative Commons Attribution Licence (http://creativecommons.org/licenses/by-sa/4.0/).

\section{Introduction}

Increasing incidence of degenerative joint disease is driving more total knee arthroplasty (TKA) procedures to improve quality of life. TKA procedures are increasing annually (i.e. $96 \%$ from 19972010 (Web ref. 1)); nevertheless, implant-centred infection remains the most common complication. Approximately $1.3 \%$ of patients receiving TKA are hospitalised for periprosthetic joint infection (PJI) within the first year of surgery (Bohm et al., 2012) - with Staphylococcus aureus (S. aureus) implicated in $67 \%$ of PJI cases (Rao et al., 2008). Of these, approximately $18 \%$ of patients experience recurrent osteolytic infections (Jämsen et al., 2009). In addition to treatment problems, revision procedures are expensive, with treatment costs reaching $\$ 75,000$ per patient (Kurtz et al., 2012).

PJI treatment commonly consists of a 2-stage revision utilising systemic intravenous antibiotics in conjunction with antibiotic-loaded bone cement (ALBC) for local antibiotic release and bone-void filling. The first stage removes the prosthesis followed by joint space debridement of infected bone and soft tissue - often leaving defects filled eventually with ALBC. In addition, joint spacers comprising moulded ALBC are placed into the joint to deliver antibiotic locally, prevent limb shortening, and in some cases preserve range of motion, typically remaining 6-8 weeks. During the second stage, the spacer is 
removed and replaced with a new implant (Goldman et al., 1996).

While revision utilising ALBC is common PJI treatment, the approach has two notable shortcomings. First, ALBC antibiotic-elution exhibits rapid, large bolus release followed by extended sub-therapeutic elution (Fletcher et al., 2004). Subtherapeutic antibiotic concentrations are identified as early as 4 weeks post-application (Jones et al., 2016), creating opportunities for antibiotic-resistant PJI development. These infections are difficult to eradicate and can lead to amputation or patient mortality. Associated difficulties arise from adherent bacteria (van de Belt et al., 2000) forming protective biofilms - impeding antibiotic penetration and producing persister colonies with delayed virulence beyond the antimicrobial temporal window of ALBC (Harms et al., 2016). The second shortcoming of revision procedures is the amount of bone lost during hardware removal and debridement. This bone loss contributes to weakening of the affected bone; thus, motivating improved treatments for eliminating infection and rapidly producing new bone.

Myriad degradable delivery systems are reported to improve PJI treatment with bone fillers over nondegradable systems such as ALBC (El-Husseiny et al., 2011), most commonly collagen fleece (El-Husseiny et al., 2011). While aiding bone formation (Reddi, 1985), collagen fleece is reported to rapidly release loaded antibiotics (95\% release in 1.5 h) (Sørensen et al., 1990). This release profile may be insufficient to eradicate PJI infections. Polyglycolide and polyL-lactide prompt bulk polymer erosion over several weeks to months to produce more clinically relevant release (El-Husseiny et al., 2011). Despite promising antibiotic release, they release acid products and require a ceramic counterpart to be osteoconductive (Campana et al., 2014). Composite devices combine multiple properties from diverse components to achieve desirable performance. Hydroxyapatite is often used in composite devices for osteoconductive properties, but can also aid antibiotic delivery (Chai et al., 2007). Composite degradable implants provide new opportunities for improving PJI treatment.

Current commercially available calcium sulphate based products (e.g. OsteoSetT ${ }^{\mathrm{TM}}$ (Wright Medical), Cerament ${ }^{\circledR} \mathrm{G}$ and V (BONESUPPORT)), are antibiotic eluting implantable beads approved for use in Europe. However, these devices do not use degradable polymers to control antibiotic release. Consequently, they release antibiotic for just 2-3 weeks. Furthermore, the rapid degradation of calcium-sulphate-based devices can lead to the formation of seromas, pockets of serous fluid with the potential to abscess (von Stechow et al., 2005).

This model of perioperative infection utilised an antibiotic-eluting bone-void filler (AEBVF) challenged against a clinically relevant infectious burden of $5 \times 10^{5}$ colony forming units (CFU) of $S$. aureus. The AEBVF used in this study comprises primarily of hydroxyapatite-coated calcium carbonate granules as the osteoconductive substrate and poly(lactideco-glycolide) (PLGA) as the ceramic binder and control for drug delivery. Tobramycin was selected for its broad-spectrum antimicrobial activity as well as its high melting point - ensuring retention of antimicrobial activity following melt moulding of the AEBVF into crouton devices.

Previous in vitro studies utilising the AEBVF (Jones et al., 2016; Brooks et al., 2015) demonstrated bactericidal activity, with the ability to eliminate $5 \times 10^{8}$ CFU of S. aureus, for 7-8 weeks (Brooks et al., 2015). These in vitro results, produced by modified Kirby-Bauer antibiotic susceptibility testing and liquid chromatography with tandem mass spectroscopy (LC/MS-MS), revealed a peak tobramycin release of $96.4 \mu \mathrm{g} / \mathrm{mL}$ at $24 \mathrm{~h}$ and a minimum of $3.77 \mu \mathrm{g} / \mathrm{mL}$ after 7 weeks of depletion in phosphate buffered saline (PBS) under sink conditions - the experimentally determined minimum bactericidal concentration was $3 \mu \mathrm{g} / \mathrm{mL}$ (Jones et al., 2016). Evaluation of LC/ MS-MS data for weeks 8-10 revealed a tobramycin concentration of $0.0 \mu \mathrm{g} / \mathrm{mL}$. In vitro evaluation of device resorption showed significant degradation after $90 \mathrm{~d}$ in PBS (Jones et al., 2016). A preliminary in vivo implant study in rabbits showed complete elimination of a bacterial challenge of $5 \times 10^{8} \mathrm{CFU}$ of S. aureus (Brooks et al., 2015). Pilot rabbit (Jones et al., 2016) and sheep [unpublished] studies supported these devices' ability to reliably form new bone during 12-week implant studies.

A statistically-powered preclinical large animal model of perioperative infection prevention was described, designed to test the ability of AEBVF resorbable composite device implants to eliminate a $S$. aureus bacterial challenge, grow new bone, and show substantial device resorption over a 12-week implantation in a sheep infection model. This AEBVF was hypothesised to:

1. prompt bone growth similarly to HA-coated calcium carbonate in a distal femoral condyle defect,

2. exhibit bactericidal activity against an $S$. aureus inoculum of $5 \times 10^{5} \mathrm{CFU}$,

3. degrade significantly after 12 weeks in situ.

This new AEBVF was evaluated in sheep, due to their similar weight and bone-remodelling rate as compared to humans. The next steps will seek to extend AEBVF device testing in an animal study modelling the two-stage revision of biofilmcontaminated total knee arthroplasty procedures.

\section{Materials and Methods}

Experimental protocols were approved by the University of Utah's Institutional Animal Care and Use Committee (Protocol\# 15-06010, approved June 2015). Twenty-eight female skeletally mature (2-4 years old) Columbia-cross sheep (60-100 kg) were divided into four groups to test the hypotheses (Table 1). Assessment of bone formation and device 
Table 1. Twelve-week study groups. "Control” groups' defects were filled with commercially available bone void filler, while both "Challenge" groups utilised the antibiotic eluting bone void filler. Only Infection groups received S. aureus inoculum.

\begin{tabular}{|l|c|c|c|c|}
\hline \multicolumn{5}{|c|}{ Experimental grouping } \\
\hline Group name & No. of sheep & Implant material & Target study duration & S. aureus 5 $\times 10^{5}$ CFU \\
\hline $\begin{array}{l}\text { Osteoconductive } \\
\text { control (OCo) }\end{array}$ & $n=7$ & ProOsteon $^{\circledR} 500 \mathrm{R}$ & 12 -weeks & $\mathbf{X}$ \\
\hline $\begin{array}{l}\text { Osteoconductive } \\
\text { challenge (OCh) }\end{array}$ & $n=7$ & AEBVF & 12 -weeks & \\
\hline $\begin{array}{l}\text { Infection } \\
\text { control (ICo) }\end{array}$ & $n=7$ & ProOsteon ${ }^{\circledR} 500 \mathrm{R}$ & 12 -weeks & \\
\hline $\begin{array}{l}\text { Infection } \\
\text { challenge (ICh) }\end{array}$ & $n=7$ & AEBVF & 12 -weeks & $\checkmark$ \\
\hline
\end{tabular}

resorption employed an osteoconductive control (OCo) consisting of commercial, clinically relevant synthetic bone-void filler ProOsteon ${ }^{\circledR 500 R}$ (CBVF; Zimmer-Biomet, Warsaw, IN, USA), as a bone-void filler standard and an osteoconductive challenge (OCh) utilising the AEBVF test devices. Both groups had 12-week endpoints to compare bone formation using metrics described below.

The AEBVF devices' antimicrobial potential was tested using a perioperative bacterial challenge that was administered to two groups: infection control (ICo) and infection challenge (ICh). The ICo utilised CBVF and bacterial inoculum $\left(5 \times 10^{5} \mathrm{CFU}\right.$ S. aureus, American Type Culture Collection (ATCC) 49230) to determine whether a positive infection signal would be generated. The ICh tested the same inoculum in AEBVF-filled defects to evaluate antimicrobial potential, osteoconduction and device resorption after 12 weeks in situ.

\section{AEBVF device fabrication}

AEBVF devices were fabricated from $350 \mathrm{mg}$ morselised and sieved (150-425 $\mu \mathrm{m})$ solid CBVF granules, $42.8 \mathrm{mg}$ polycaprolactone (PCL, average molecular weight $\left(\mathrm{M}_{\mathrm{w}}\right) \sim 10 \mathrm{kDa}$, Sigma-Aldrich, St. Louis, MO, USA), $21.4 \mathrm{mg}$ polyethylene glycol (PEG, average $\mathrm{M}_{\mathrm{w}} \sim 20 \mathrm{kDa}$, Sigma-Aldrich, USA), $85.5 \mathrm{mg}$ PLGA 50 : 50 ratio lactide-co-glycolide, inherent viscosity 0.55-0.75 dL/g (Durect, Cupertino, CA, USA), $22.2 \mathrm{mg}$ calcium chloride (particle size $<425 \mu \mathrm{m}$ ) and $85.5 \mathrm{mg}$ solid tobramycin powder (Research Products International, Mt. Prospect, IL, USA). PLGA was dissolved in acetone $(0.21 \mathrm{mg} /$ $\mathrm{mL})$. PEG and PCL were melted at $75^{\circ} \mathrm{C}$ and stirred as CBVF, calcium chloride, and tobramycin solids were added sequentially. After achieving a uniform mixture, PLGA solution was added and mixed long enough to evaporate the acetone (30-45 s). The warm mixture was pressed into moulds $(6 \times 2 \times 2 \mathrm{~mm})$, cooled to room temperature $\left(20-25^{\circ} \mathrm{C}\right)$. Once cool, the croutons were rolled in hot $\left(70^{\circ} \mathrm{C}\right) \mathrm{CBVF}(150-$ $425 \mu \mathrm{m}$ ) to produce an outer crust that covered the glassy polymeric surface to theoretically increase the device's osteoconductivity - giving final dimensions of $\sim 7 \times 3 \times 3 \mathrm{~mm}$ ). Finished devices were then stored at $-80{ }^{\circ} \mathrm{C}$ to preserve polymers and tobramycin antibiotic capacity. Prior to surgical implantation, the devices were sterilised using Gamma irradiation.

To ensure that all of the solvent had flashed off of the devices during fabrication, gas chromatography was performed to quantify residual solvent.

\section{Inoculum preparation}

S. aureus inoculum created from ATCC 49230 strain (An et al., 2006) cultured on Tripticase soy agar (TSA) (Thermo Scientific, Waltham, MA, USA) was incubated at $37^{\circ} \mathrm{C}$ overnight. Colonies were added to PBS (Thermo Scientific, USA) to achieve a $0.5 \mathrm{McF}$ arland (Jones et al., 2016) and then diluted $(1: 150)$ with PBS to achieve $5 \times 10^{5} \mathrm{CFU} / \mathrm{mL}$. Aliquots $(1 \mathrm{~mL})$ were pelleted at $25,200 \times \mathrm{g}$ for $10 \mathrm{~min}$ and resuspended in $30 \mu \mathrm{L}$ PBS. Final concentration of $5 \times 10^{5}$ $\mathrm{CFU} / \mathrm{mL}$ was verified by culturing serially diluted sample inocula on TSA according to Clinical and Laboratory Standards Institute guidelines (Clinical and Laboratory Standards Institute, 2006).

\section{Surgical procedure}

Procedures followed those performed by Sinclair $e t$ al. (2013). Prior to surgery, sheep were anaesthetised using propofol $(5-10 \mathrm{mg} / \mathrm{kg})$. The medial knee joint and surrounding area on the right hind leg were shaved and surgically prepped. After sterile draping, an incision starting $30 \mathrm{~mm}$ proximal and just $(\sim 10 \mathrm{~mm})$ anterior to the medial collateral ligament (MCL) was made, extending down and parallel to the MCL another $30 \mathrm{~mm}$. Incision depth was just above the cortical bone surface. After incision and periosteum displacement, a defect was created anterior to the MCL with dimensions of $7 \mathrm{~mm}$ tall, $9 \mathrm{~mm}$ wide, and $7.5 \mathrm{~mm}$ deep using a custom sizing tool and a 1-mm drill bit to hollow out 4 corner holes outlining the defect perimeter. An osteotome was then used to chisel the defect to specified dimensions. In both control experiments, approximately $1.5 \mathrm{~mL}(\sim 500 \mathrm{mg})$ of $C B V F$, as received (1-5 mm), was packed in with a tamper. In both challenge experiments, six moulded AEBVF devices $(\sim 0.378 \mathrm{~mL})$ were stacked into the defect, filling the volume (Fig. 1). After each defect was loaded in the ICh and ICo, $30 \mu \mathrm{L}$ of $S$. aureus inoculum $\left(5 \times 10^{5} \mathrm{CFU}\right)$ was dispensed into the defect, into the space between the croutons, using a $200 \mu \mathrm{L}$ 

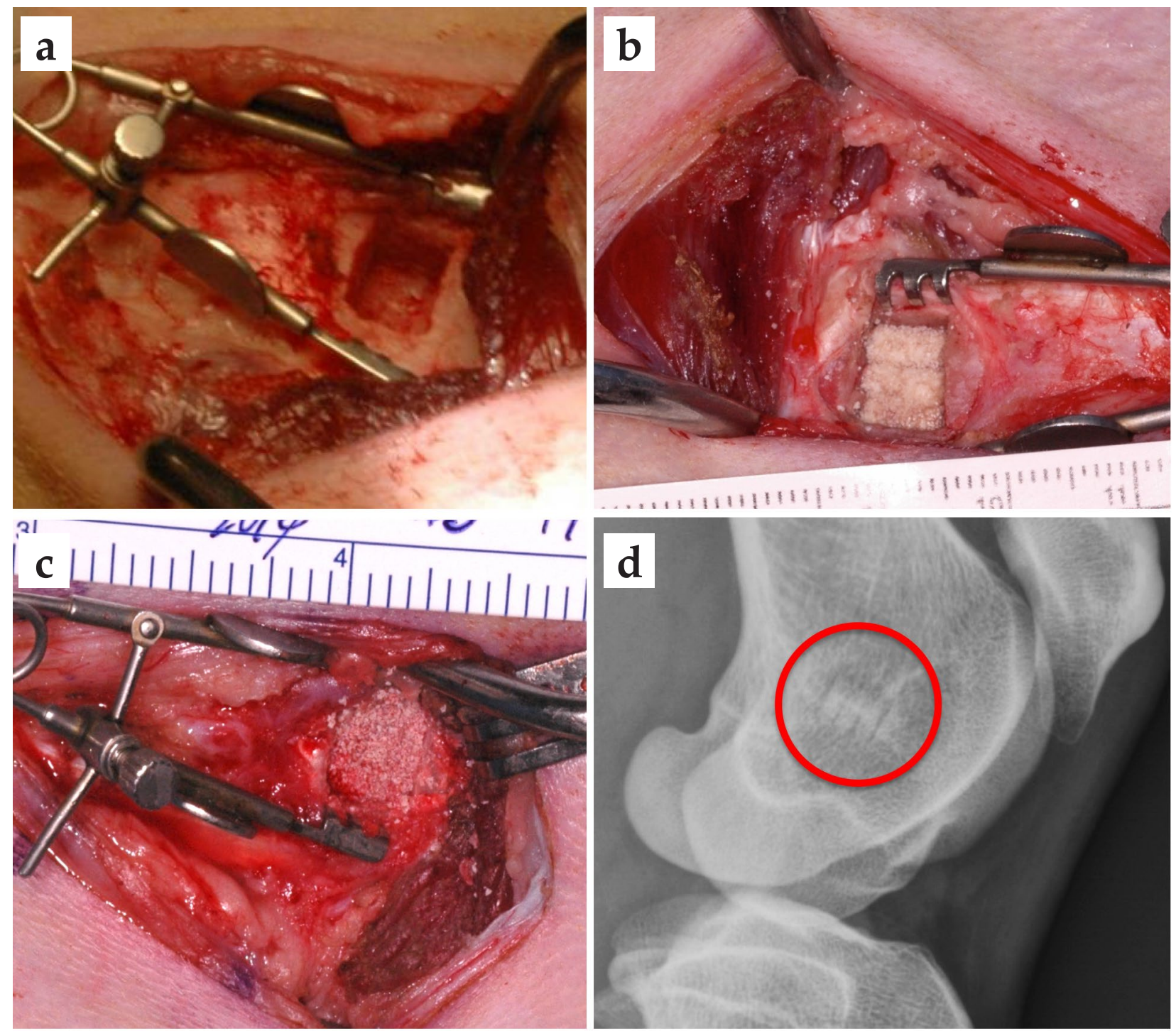

Fig. 1. Surgical images. Showing a) the empty, surgically created defect, b) the defect filled with AEBVF, c) the defect filled with CBVF, and d) radiograph showing the location of the surgically created defect on the medial face of the distal femoral condyle. The red circle shows the rectangular defect and the antibiotic eluting bone void filler devices that were used to fill the defect.

pipette and a sterile pipette tip. The inoculum was added after the croutons were in position, rather than prior, to prevent inadvertent contamination of the surgical field. A cortical bone cap was then secured with bone wax on the defect and the surgical site was sutured closed in layers. Subcutaneous closure used 3-0 Vicryl suture (Ethicon, Somerville, NJ, USA), followed by 3-0 Prolene sutures (Ethicon, USA) for cutaneous closure. The surgical site was cleaned with sterile saline and betadine solution and then dressed.

\section{Systemic tobramycin concentration}

Tobramycin concentrations, systemised from implant release to sheep blood circulation, were measured using the clinical standard immunoassay in a CLIAcertified outsourced facility (ARUP assay "0090270 tobramycin", Abbott FPIA Immunoassay; Architect ci8200, ARUP, Salt Lake City, USA). Reportable assay tobramycin detection range was $0.4-40 \mu \mathrm{g} / \mathrm{mL}$. Commercial sheep plasma (BioChemed Services,
Winchester, VA, USA, 032-APSC-MP, heparinised pooled, male) was used to generate standard curves using spiked tobramycin. Sheep blood was drawn at 24, 48, $96 \mathrm{~h}$ and in some cases time of euthanasia, from the jugular vein into heparinised tubes and immediately centrifuged to produce platelet poor plasma, and stored in plastic Eppendorf vials at $-20{ }^{\circ} \mathrm{C}$ until thawed for assay. A total of 78 systemic blood draws ( $n=2$ draws, $n=3$ assays of each).

\section{Necropsy}

Sheep were euthanised at the study's 12-week endpoint or earlier if they presented with Grade 3 or higher symptoms from the modified Checketts scoring (Sinclair et al., 2013; Checketts et al., 2000):

- Grade 1: inflammation and redness at the incision site. Standard response to operative trauma: no treatment or sacrifice. No positive culture.

- Grade 2: Inflammation and redness, regions of incision site not closed, swab for microbiology 
assessment and continue to monitor. No limping, sheep eating, not irritable. Possible colony growth: no sacrifice, watch carefully.

- Grade 3: Inflammation and redness, regions of incision showing pus, limping, local tenderness, microbiological analysis confirms infection with significant growth in cultures, irritable behaviour, off feed: animal to be sacrificed.

- Grade 4: Inflammation, incision site completely open, pus, implant exposure, limping, local tenderness, off feed, lethargy (systemic sign of infection), high temperature: sacrifice immediately).

At the time of necropsy, surgical defects were created in the contralateral limb and a time " 0 " specimen, matching the ipsilateral limb, implanted.

\section{Microbiology}

Microbiological analysis was performed as previously reported (Sinclair et al., 2013). At necropsy, three swab samples were collected from the: subcutaneous space, intramuscular trans-section, and bone surface. Aseptic technique was used to obtain $\sim 1 \mathrm{~g}$ of soft tissue from the aforementioned locations, including the muscle in direct apposition with the surgically created defect - placed in 10-mL vials of sterile PBS. Swabs were agitated in $5 \mathrm{~mL}$ of PBS and sonicated for $5 \mathrm{~min}$. After sonication, $100 \mu \mathrm{L}$ aliquots were diluted into $900 \mu \mathrm{L}$ of cation-adjusted Mueller Hinton Broth (Becton Dickinson, Murry, UT, USA) and serially diluted through 7 iterations. From each dilution, $10 \mu \mathrm{L}$ aliquots were plated in triplicate on TSA for enumeration. Each tissue sample was macerated in a conical tube tissue grinder, sonicated for $10 \mathrm{~min}$, and spun centrifugally for $2 \mathrm{~min}$ at 2,100 $\times g$ to separate the tissue from the media. Aliquots $(100 \mu \mathrm{L})$ from each sample container were serially diluted and cultured as already described in inoculum preparation (above).

\section{$\mu-C T$ analysis}

The harvested distal femurs were scanned using a $\mu$-CT (Quantun FX, Perkin Elmer, Waltham, MA, USA). The $\mu-C T$ scans were acquired using a tube voltage of $90 \mathrm{kV}$, tube current $180 \mu \mathrm{A}$ and a fieldof-view (FOV) of $20 \mathrm{~mm}$ which resulted in $\sim 40 \mu \mathrm{m}$ slices. Following imaging, the scans were used to assess the estimated percentage of bone and residual implant within the defect. To do so, the images were loaded into Amira analysis software. Any soft tissue remaining following dissection was thresholded away by way of grey scale analysis, leaving only bone. Due to the slight variability of the defect size, a uniform region of interest (ROI) was created by using scans from time 0 specimens, ensuring measurements between groups were consistent. This best fit ROI allowed for an $\sim 7.5 \times 7.5 \mathrm{~mm}$ cylinder to be extracted from the central region of the defect, which equalled $\sim 340 \mathrm{~mm}^{2}$ of area. The CBVF and AEBVF devices were also removed by way of grey-scale thresholding to allow for the estimated percentage of bone within the defect to be measured.

\section{Histomorphometric measurements}

Prior to $12^{\text {th }}$-week necropsy, sheep in groups 1,2 , and 4 were injected with calcein $(10-20 \mathrm{mg} / \mathrm{kg}) 16$ and $5 \mathrm{~d}$ prior to necropsy to fluorescently label new bone (Sinclair et al., 2013). Following necropsy, the implant and surrounding bone were fixed in $10 \%$ neutral buffered formalin (NBF), dehydrated by ascending ethanol concentrations, and embedded in poly(methyl methacrylate) (PMMA) (Sinclair et al., 2013). Embedded samples were cut into three $\sim 2 \mathrm{~mm}$ sections, then ground and polished for scanning electron microscopy (SEM) and backscatter SEM (BSE). Time " 0 " specimen were used for baseline data to evaluate: the effects of the surgical trauma, defect margins for 12-week sample comparison, and device packing within the defect. BSE images were qualitatively analysed for bone resorption, bone remodelling, porosity, woven bone formation, and bone formation within implant-containing regions (Jones et al., 2016).

Following BSE, implant containing PMMA sections with surrounding tissue were ground to optical transparency (50-70 $\mu \mathrm{m}$ thickness). Fluorescence microscopy and BIOQUANT image analysis (BIOQUANT Corporation, Nashville, TN, USA) software were used to quantify bone mineral apposition (MAR, $\mu \mathrm{m} /$ day) at various bone depths along implant margins (Jones et al., 2016; Sinclair et al., 2013).

Finally, histological sections were stained with Sanderson's rapid bone stain (SRBS) to provide semi-quantitative analysis of bone/implant interfacial bioactivity, i.e. acute and chronic intraosseous inflammation, periosteal inflammation and bone necrosis as described previously (Jones et al., 2016; Brooks et al., 2015; Sinclair et al., 2013). Implant biocompatibility was evaluated using bone presence and quality, fibrous tissue presence, and inflammatory cells at bone-implant interfaces. Histology was scored (scale of 0 to 4 ) by three independent and blinded investigators using light microscopy for:

1. capsule thickness $(4=<50 \mu \mathrm{m} ; 0=>250 \mu \mathrm{m})$,

2. inflammatory response $(4=$ no inflammatory response; $0=$ moderate to severe inflammation with abscess),

3. cellular infiltration $(4=$ mostly bone; $0=$ dense tissue and exclusively inflammatory cells),

4. qualitative bone reaction $(4=$ similar appearance to host bone; 0 = dead bone) according to previously used histomorphological scoring metrics (Brooks et al., 2015; Sinclair et al., 2013).

All scores from a single cohort were averaged for each metric.

\section{Statistical analysis}

Group size determination assumed that all sheep receiving AEBVF groups would make it to the 12week end point with a zero confirmed infection incidence. It was assumed that all sheep in the ICo group would have confirmed infection; thus, requiring euthanasia prior to week 6 . Under these 
assumptions (0/7 vs. 7/7 infected), the sample size of $n=7$ per group provided $>99 \%$ power, using a two-sided alpha 0.05 comparison. Even if the proportions were closer together (1/7 vs. 6/7), which were the expected proportions for the Grade 3 or higher inflammation outcome, the results of a twosided Fisher's exact test would still yield a statistically significant difference. These power analyses were based on simple comparisons of proportions. The logrank test, which takes time to event into account, would have even greater statistical power than these conservative computations.

Mean and variance for experimental populations were used to demonstrate reproducibility. Survival analysis, means comparisons using Tukey-Kramer HSD Confidence Quantile followed by one-way analysis of variance were used to determine statistical significance between experimental and control groups for SRBS- and MAR-stained slides. Significant differences were asserted for $p$-values $<0.05$. In vivo animal data were evaluated using survival analysis.

\section{Results}

\section{Device fabrication}

Implants created in 11-device batches exhibited measured dimensions of $6.0 \pm 0.3 \mathrm{~mm}$ tall, $2.1 \pm 0.2 \mathrm{~mm}$ deep, and $1.9 \pm 0.2 \mathrm{~mm}$ wide. Average device mass was $44.7 \pm 5.8 \mathrm{mg}$ and average tobramycin content was $9.5 \%$. Gas chromatography revealed no residual solvent within the fabricated devices.

CBVF was used as received from the manufacturer with a size distribution of $1-5 \mathrm{~mm}$.

\section{Microbiology}

Bacterial inocula for infection group surgeries were verified pre- and post-inoculation at $5 \times 10^{5} \mathrm{CFU}$. Culturing of tissues and tissue swabs revealed no detectable bacterial growth in the OCh or ICh samples at the 12-week endpoint. Bacteria in ICo were observed throughout the tissue bed extending from subcutaneous spaces into bone, with bacteria in bone measured consistently above $5 \times 10^{4} \mathrm{CFU} / \mathrm{g}$.

\section{Systemic tobramycin concentration}

All blood assays from all time points (i.e. at 24, 48, $96 \mathrm{~h}$ post-implantation and also a limited number of samples at sheep sacrifice) showed no detectable tobramycin in sheep blood by this clinical assay FPIA method. All assays were below the limit of detection $(0.4 \mu \mathrm{g} / \mathrm{mL})$, indicating no detectable systemisation of tobramycin locally released to tissue from the implanted devices.

\section{Necropsy}

Due to the rapid onset of infection, clinical infection indicators consistent with Grade 3 on the modified Checketts score (Sinclair et al., 2013; Checketts et al., 2000), and veterinary recommendation, all ICo animals were sacrificed 6-11 d post-surgery. All other groups were euthanised at the scheduled 12-week endpoint. Necropsies of ICh, OCo and OCh animals revealed healthy tissue extending from skin to bone.

\section{$\mu-C T$ analysis}

The OCo and ICh groups' defects showed nearly equivalent bone formation rates $(35.54 \pm 4.16 \%$ and $35.52 \pm 13.43 \%$ ), respectively (Fig. 2), while bone formation in the OCh was slightly lower at $32.89 \pm 7.81 \%$. The ICo had the least bone formation with a new bone area of $4.30 \pm 1.96 \%$ ).

Residual implant analysis of time " 0 " sections showed a mean implant percentage of $57.29 \pm 1.45$ for the AEBVF and $46.55 \pm 4.39$ for the CBVF devices. Evaluation of 12-week data showed residual implant of $2.47 \pm 1.87 \%$ for the AEBVF and $35.55 \pm 2.87 \%$ for the CBVF devices (Fig. 3).

\section{BSE image analysis}

Surgical defects from OCo, OCh, and ICh showed bone formation throughout defects (Fig. 4). Within these groups' defects, the BVF was integrated with newly formed bone. BSE evidence revealed osteoblasts, shown in low and higher magnification images (Fig. 4) of the OCh. Negligible bone formation was observed in ICo only surviving 6-11 d postimplantation. Within this group, extensive host bone resorption was observed surrounding the defect;

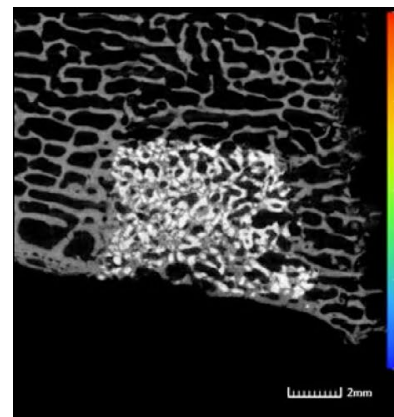

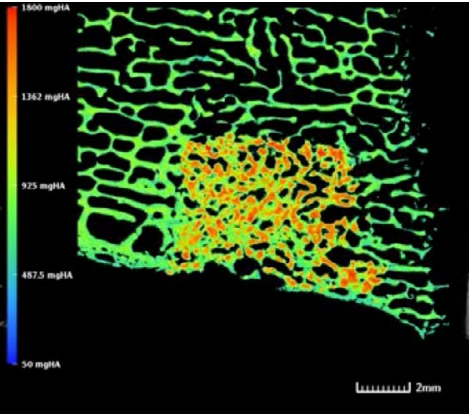

Osteoconductive control

(CBVF) - 12 weeks

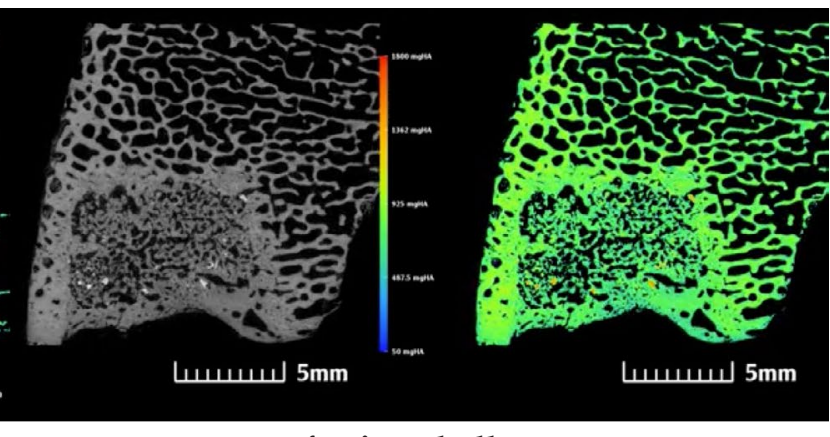

Infection challenge

Fig. 2. $\mu-C T$ image comparison. Twelve-week $\mu-C T$ analysis showed similar void filling capacity of the CBVF osteoconductive control and AEBVF infection challenge devices - despite the bacterial challenge. Images have been coloured to show new bone (green) and residual implant (red-orange). 
consistent with chronic osteomyelitis. This positive osteomyelitis signal supported the known bacterial virulence of human strain ATCC 49230 and absence of its deleterious pathology in the AEBVF group.

\section{Mineral apposition rate analysis}

Actively remodelling bone was observed in ICh, OCo and Och samples (Fig. 5), evidenced by fluorescing Calcein signals in bone (Fig. 5a,b). Importantly, all sections exhibited accelerated MAR (mean $\sim 1.4 \mu \mathrm{m} / \mathrm{d}$ ), roughly doubling normal boneremodelling rates in sheep $(0.71 \pm 0.21 \mu \mathrm{m} / \mathrm{d})$ (Brooks et al., 2015). This finding supports the hypothesis that new bone formation is not adversely affected by local, extended tobramycin release or BVF resorption. Bone formation observed in all three groups was not statistically different $(p>0.05)$ for both perimeter and centre of defect MAR measurements (Fig. 5c).

\section{SRBS - light microscopy}

Light microscopy corroborated findings from BVF implant BSE and MAR analyses. Bone formation throughout surgical defects and integration of morselised CBVF granules was observed in ICh, OCo and OCh (Fig. 6) groups. Active bone formation, characterised by osteoclastic resorption (Fig. 7a- d), coupled with osteoid deposition was observed throughout cohort defect sites. Consistent with previous results from pilot rabbit (Jones et al., 2016) and AEBVF sheep studies (unpublished), a mild inflammatory response was present, but only observed adjacent to residual polymer and CBVF (Fig. $5 \mathbf{e}, \mathbf{f})$, where macrophages were largely concentrated.

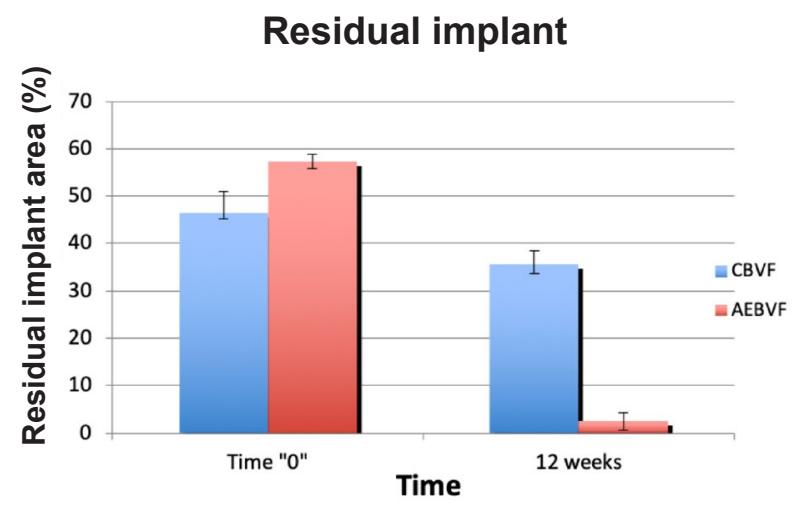

Fig. 3. Residual implant analysis. After 12-weeks in situ CBVF osteoconductive control devices showed a residual implant percentage of $35.55 \pm 2.87$, while AEBVF osteoconductive challenge devices showed a mere $2.47 \pm 1.87 \%$.

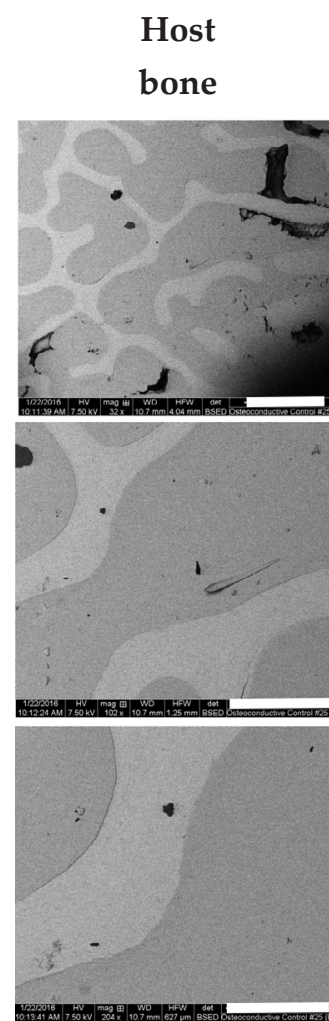

\section{Osteoconductive control (OCo)}
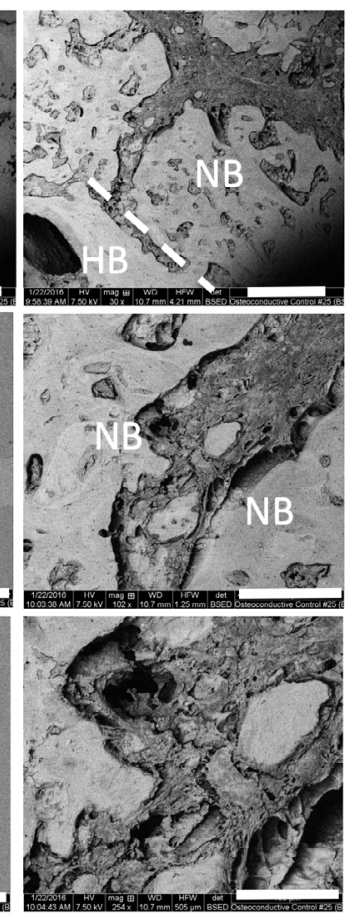

Osteoconductive challenge (OCh)
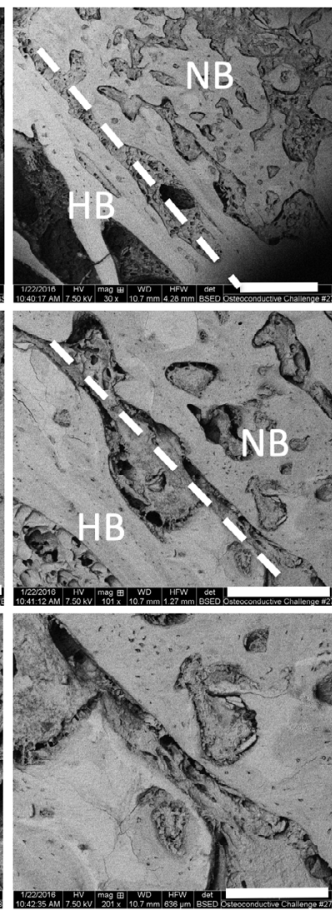

Infection control (ICo)
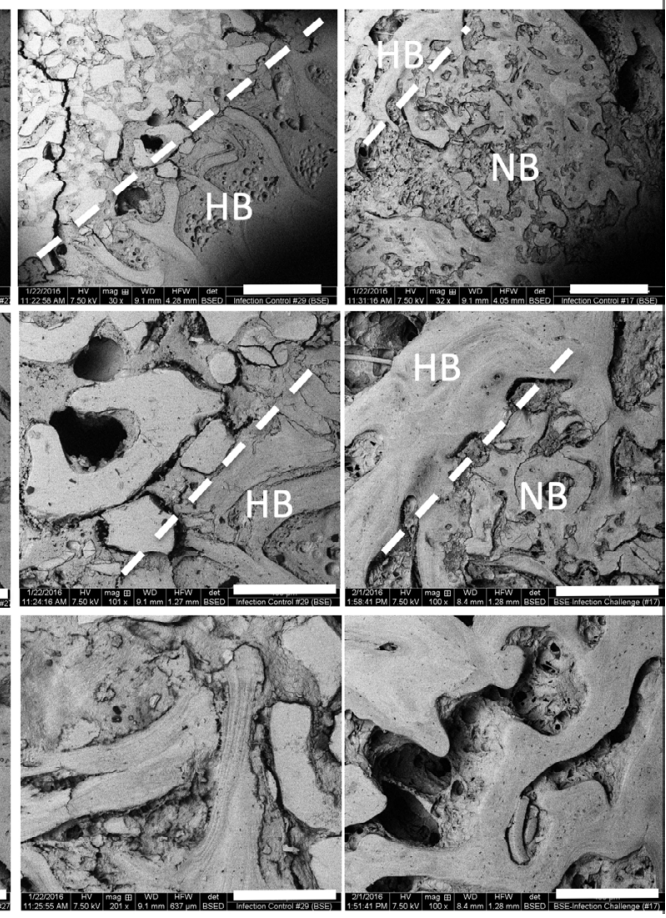

Infection challenge (ICh)

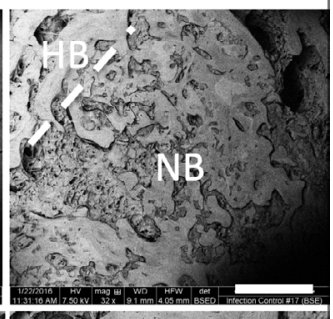

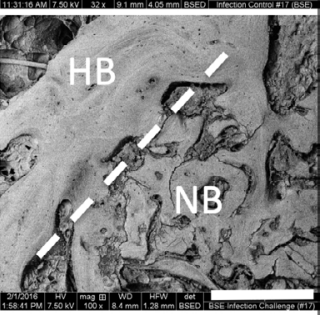

Fig. 4. Backscattered electron imaging. Comparison of host bone (Column 1) with the CBVF osteoconductive control (Column 2), AEBVF osteoconductive challenge (Column 3), CBVF infection control (Column 4), and AEBVF infection challenge (Column 5) using BSE. BSE allowed easy distinction of newly formed bone ("NB" - darker shade of grey) from the older host bone (HB) and implanted material (lighter shade of grey). Columns 2, 3 and 5 show new bone formation in implant sites after 12 weeks in situ. Images (Row 1, Scale bar $=1 \mathrm{~mm}$ ) show bone formation within defects, with comparable bone formation in Columns 2, 3 and 5 . Notably, no bone formation was observed in Column 4, infection control, euthanised 6-11 d post-op due to unresolved infection. In contrast, higher magnification images (Row 2, Scale bar $=400 \mu \mathrm{m}$ and Row 3, Scale bar $=200 \mu \mathrm{m}$ ) reveal resorption of existing host bone. 


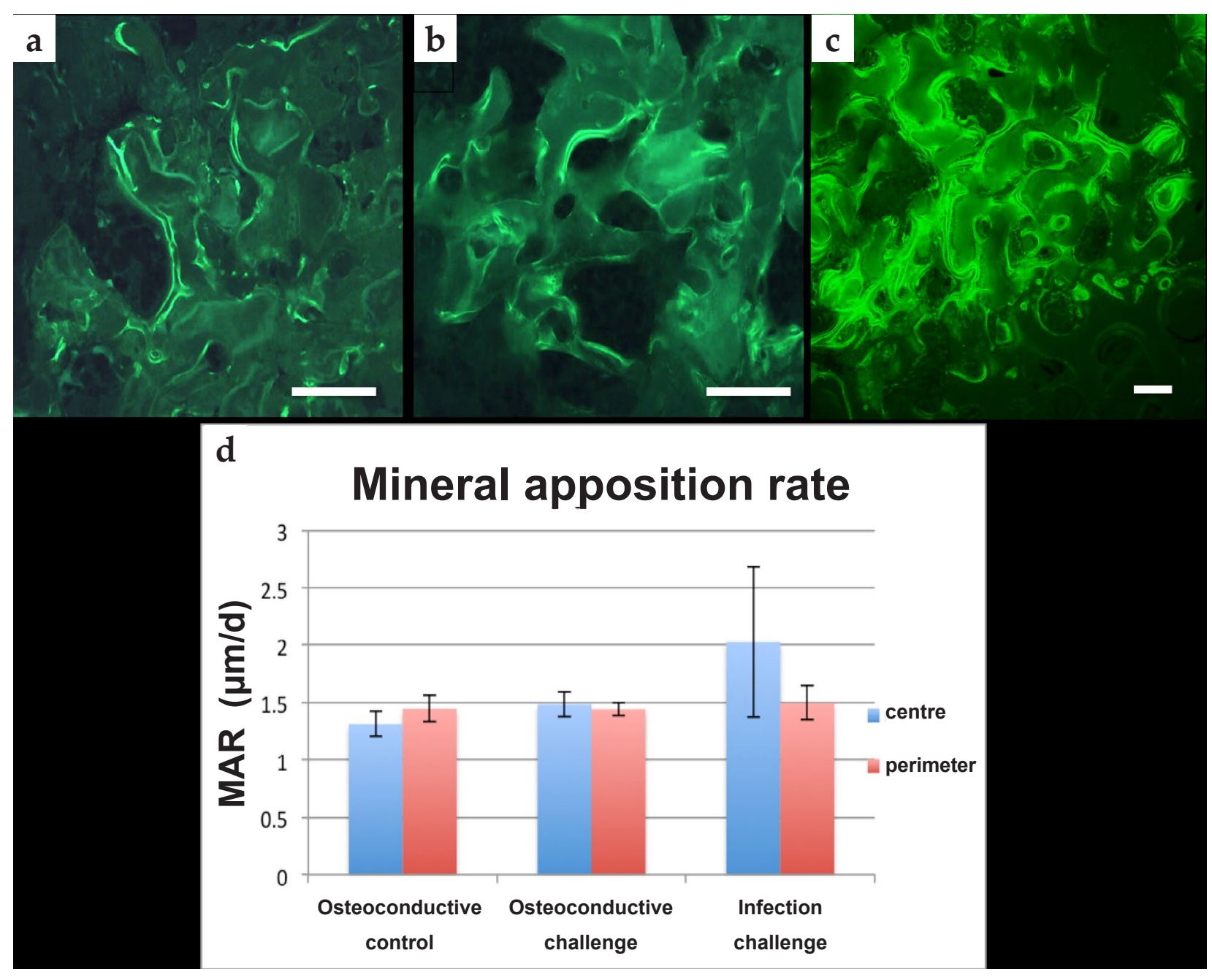

Fig. 5 Mineral apposition analysis from 4 study groups. Fluorescence microscopy showed mineralising surfaces using calcein labels (Scale bar $=250 \mu \mathrm{m}$ ) in surgical defects for: a) Osteoconductive challenge, b) Infection challenge and c) Osteoconductive control groups. MAR comparisons of AEBVFs. d) Centres and perimeters revealed no statistical differences $(p>0.05)$ between any calcein-labelled groups (infection challenge, osteoconductive challenge and control).

ICo animals not only survived a mere 6-11 d, exhibiting positive infection signs confirmed by microbiology, but these animals also showed severe inflammatory responses consistent with osteomyelitis. Numerous inflammatory cells, including neutrophils and macrophages, characterised this signal; as well as numerous osteoclasts and extensive osteoclastic resorption (Fig. 5g,h).

Semi-quantitative histomorphometric analysis of the defect perimeter revealed no acute intraosseous inflammation and minimal to no chronic intraosseous (0.4) or periosteal (0.8) inflammation in the OCo. Similar results were observed with the $\mathrm{OCh}$; however, minimal-to-mild inflammation (1.2) was observed in the periosteum. Minimal-to-mild inflammation was also observed in the ICh, with chronic intraosseous and periosteal inflammation scores of 1.0 and 1.6. In contrast, the ICo showed moderate-to-severe scores for acute, chronic and periosteal inflammation, as well as bone necrosis with scores of 3.0, 2.6, 2.2, and 4.0; respectively.
Evaluation of the defect centre generally produced lower scores, with normal tissue observed for the OCo and OCh. Minimal chronic intraosseous inflammation was observed in the ICh with a score of 0.4. Consistent with the perimeter evaluations of the ICo, high scores were also observed for the defect centre with scores of 3.4, 2.4 and 3.2 being recorded for acute and chronic intraosseous inflammation, and bone necrosis.

\section{Discussion}

Perioperative infection prevention remains an unmet need for patients and increasing challenge for clinicians and surgeons. Perioperative infection and PJI treatment may have limited efficacy due to bone loss and insufficient antibiotic release (van de Belt et al., 2000; Checketts, 2000). This study evaluated a resorbable AEBVF's ability to form new bone, provide relevant antibiotic delivery with therapeutic 


\section{Time "0"}

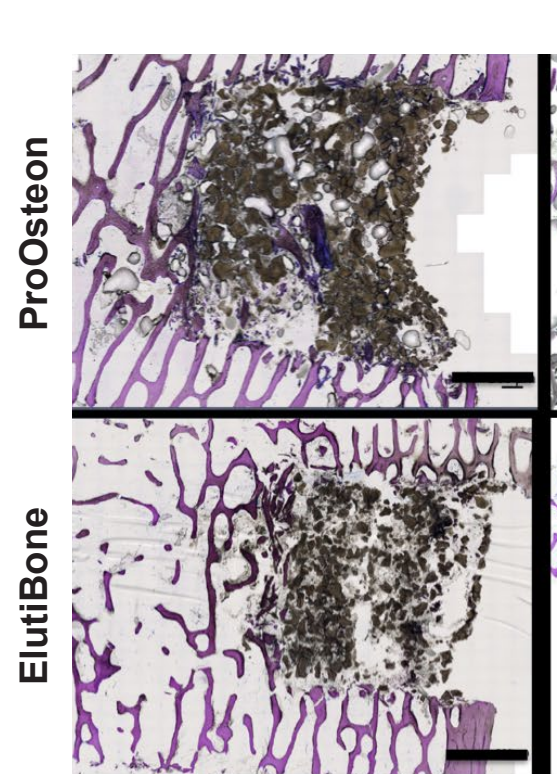

Time "0"
Osteo control

12 weeks

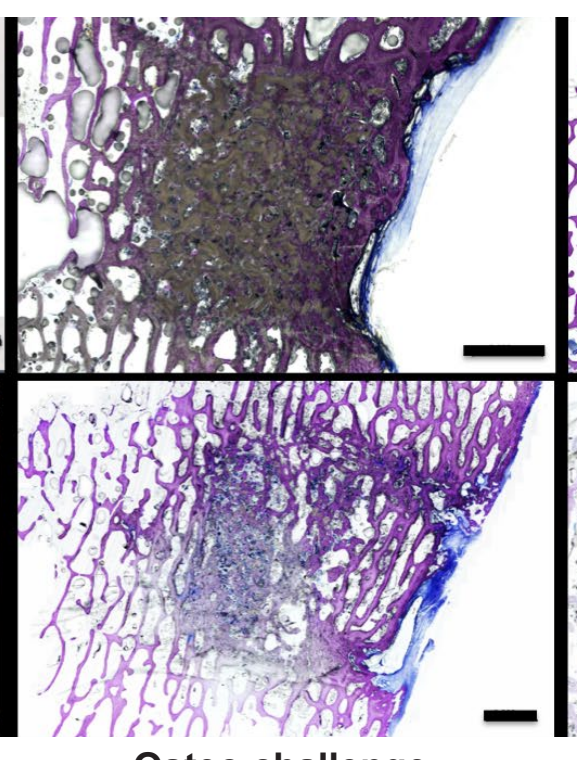

Osteo challenge

12 weeks
Infection control

6-11 d
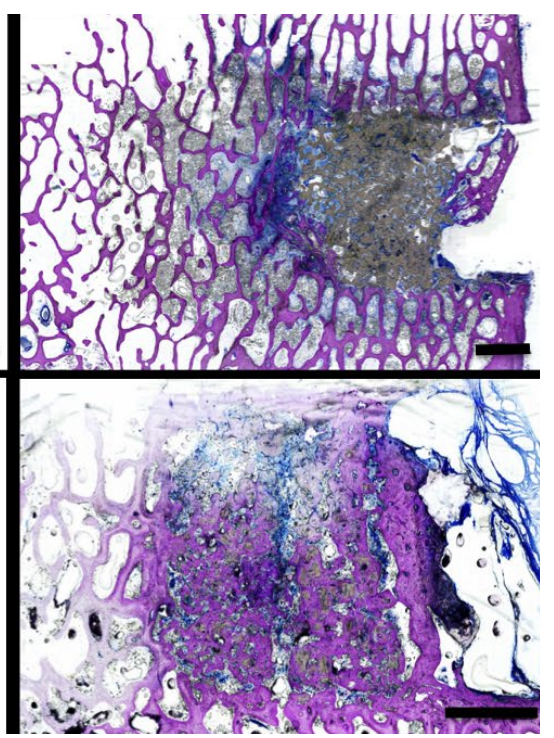

Infection challenge

12 weeks

Fig. 6. Microscopy comparisons of BVF implants. Column 1: Time "0" (left) control CBVF granules (top) and AEBVF test implants (bottom), Column 2: 12-week images evaluating the BVF's osteoconductive capacity (CBVF control - top; AEBVF test - bottom), and Column 3: Antimicrobial potential (CBVF control + S. aureus - top; AEBVF test + S. aureus - bottom). Extensive bone formation was seen in the CBVF osteoconductive control and AEBVF challenge, as well as the infection challenge - despite injection of $5 \times 10^{5} \mathrm{CFU}$ S. aureus. Scale bar $=2 \mathrm{~mm}$.

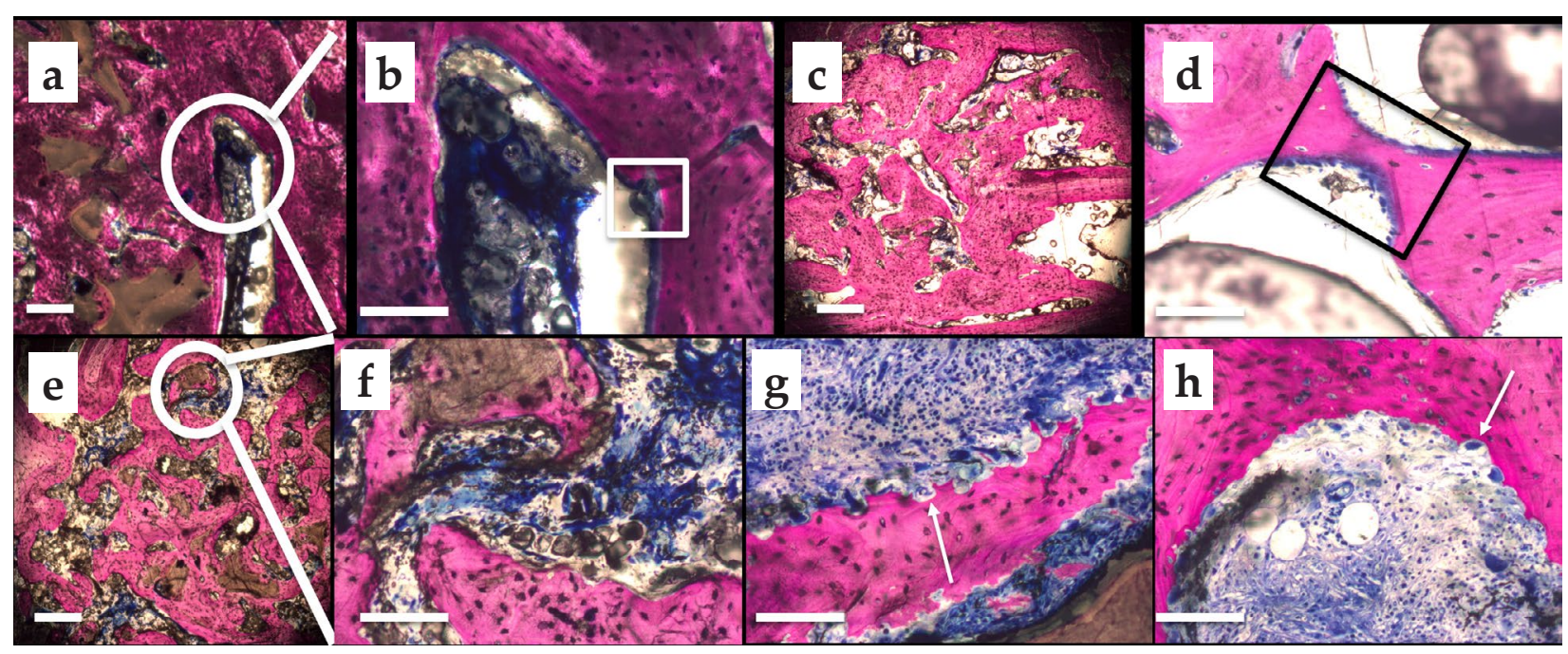

Fig. 7. Sanderson's rapid bone staining of the 4 study groups. SRBS confirmed the extensive bone formation that was observed with BSE and MAR in osteoconductive controls (CBVF). a) All bone left of the void and circled region of interest, is newly formed bone within the centre of the surgically created defect. Right of the void is host bone. (Scale bar $=500 \mu \mathrm{m}$ ) Also shown is new bone formation encasing implanted CBVF material, and active new bone formation, characterised by presence of $\mathbf{b}$ ) osteoclasts-bone resorbing cells within the square area of interest, osteoblasts-bone depositing cells in lines leading up to the osteoclasts, and osteoidthe new unmineralised bone matrix (blue) in which the osteoblasts are embedded, was observed throughout defects (Scale bar $=200 \mu \mathrm{m}$ ). Similar to osteoconductive controls, c) new bone was observed to fill the surgically created defects (Scale bar $=500 \mu \mathrm{m}$ ) of osteoconductive challenge sites where $\mathbf{d}$ ) active bone formation was also observed (rectangular area of interest; Scale bar $=200 \mu \mathrm{m}$ ). Infection challenge (AEBVF): e) Extensive new bone formation filling the surgically created defect (Scale bar $=500 \mu \mathrm{m}$ ) with $\mathbf{f}$ ) localised inflammatory response centred around residual ceramic material (circulating macrophages $=$ dark blue spheres; Scale bar $=200 \mu \mathrm{m}$ ) was observed in infection challenge sites at 12-week endpoints. Infection controls required euthanasia 6-11 d post-implantation. SRBS revealed positive infection signals (chronic inflammatory responses (macrophages = dark blue spheres, neutrophils = light blue spheres) and extensive osteoclastic bone resorption) observed in $\mathbf{g}$ and $\mathbf{h}$ (arrows point to osteoclastic resorption pits; Scale bar $=200 \mu \mathrm{m}$ ). 
concentrations, and resorb in a large animal defect model. Hypotheses addressed were that this AEBVF:

1. demonstrated osteoconductivity in a distal femoral condyle defect,

2. eliminated relevant $S$. aureus contamination of $5 \times 10^{5} \mathrm{CFU}$,

3. degraded significantly after 12 weeks in situ.

With continued research and validation, the AEBVF described in this study has the potential to be utilised during the first stage of a revision joint replacement, in conjunction with antibiotic loaded bone cement, to prevent recurrent infection and facilitate new bone formation during the 6-8 week hardware-exchange window.

AEBVF osteoconductivity (Hypothesis 1) was supported by bone formation and extensive bone remodelling within surgical defects in ICh, OCo and OCh groups. Histomorphometric analysis showed substantial bone formation in all groups except ICo (Fig. 5). OCo and ICh group defects showed nearly equivalent bone formation $(35.54 \pm 4.16 \%$ and $35.52 \pm 13.43 \%$ ), respectively, while bone formation in OCh sites was slightly lower (32.89 $\pm 7.81 \%)$, but differences were not statistically significant. ICo had the least bone formation ( $4.30 \pm 1.96 \%)$, as expected for required 6-11 d necropsy. In this model, AEBVF facilitated new bone formation within defects similar to clinically used CBVF, ProOsteon ${ }^{\circledR 500 R}$. Greater MAR and less bone in defects containing CBVF may be caused by slower implant resorption compared to AEBVF, delaying wound healing responses. A previous study showed increased resorption of a composite device comprising PCL with tri-calcium phosphate, facilitating bone formation (Miyai et al., 2008). While increased degradation promotes bone formation, others showed that implant resorption faster than the bone apposition rate might limit bone formation (Hing et al., 2007). Another explanation for increased bone formation may be increased hydroxyapatite surface area. The CBVF hydroxyapatite shell becomes exposed as the more quickly degrading calcium carbonate core is resorbed. Increased hydroxyapatite surface area correlates with increased osteoconductivity of synthetic bone grafts (Boyde et al., 1999).

Rapid device degradation and greater bone formation were also observed with MAR and SRBS. However, these differences were not statistically significant $(p>0.05)$ from the OCo, OCh and ICh groups. However, unlike the CBVF, the AEBVF provides therapeutic antibiotic concentrations to mitigate bone-centred infections.

Infection prevention following $S$. aureus contamination $\left(5 \times 10^{5} \mathrm{CFU}\right)$ was achieved in the ICh; supporting AEBVF bactericidal capacity and Hypothesis 2. Microbiological analysis of swabs and tissue biopsies showed no bacteria in tissue surrounding defects in ICh sites at 12-week endpoints. Active bone remodelling was also evident with similar MAR to osteoconductive groups (Fig. 3c,d).

ICo animals exhibited clinical signs of infection (Fletcher et al., 2004) 6-11 d post-implantation, requiring early euthanasia. Microbiological analysis revealed average bacterial counts of $5 \times 10^{4} \mathrm{CFU} / \mathrm{g}$ in the infected tissue samples. SRBS displayed chronic osteomyelitis (Fig. $\mathbf{5 g}$,h) characterised by areas of "moth-eaten" bone. Clinical evidence and bacterial counts of ICo animals supported the virulence of the clinically relevant $S$. aureus isolate commonly used to study osteomyelitis (Reizner et al., 2014). Bone samples were not collected for this work due to the fact that in the previous pilot study:

1. contamination of the overlying soft tissue would contaminate the bone surface producing unreliable bacterial counts,

2. in an effort to preserve the defect for histomorphometric analysis, the core was too far removed $(\sim 6 \mathrm{~mm})$ from the surgical defect to assess contamination within the defect,

3. radiographic and histomorphometric evaluation reliably identifies osteomyelitis $10 \mathrm{~d}$ post-op due to the bone's moth-eaten appearance.

AEBVF in ICh defects prevented infection with identical $S$. aureus inocula, asserting effective AEBVF bactericidal capacity, consistent with success previously observed with this AEBVF in a rabbit infection model (Jones et al., 2016) eliminating $S$. aureus contamination of $5 \times 10^{7}$ CFU. Combination devices have been studied with delivery mechanisms that release therapeutic antibiotic doses against S. aureus (Brooks et al., 2015; Sinclair et al., 2013). While these systems are bactericidal against common pathogens, few have clinically relevant 6-8 week elution above the minimum bactericidal concentration (MBC) (Jones et al., 2016). This duration has proven necessary for eradication of persister bacteria (Harms et al., 2016) and coincides with the 6-8 week exchange window common to two-stage revision procedures.

AEBVF degradation (Hypothesis 3) was supported by less residual BVF after 12 weeks in situ in the OCh and ICh groups. In contrast, little-to-no device degradation was observed with both OCo and ICo groups (Figures 2,4). Control group (CBVF, ProOsteon $\AA 500 R$ ) degradation was similar to other results describing incomplete degradation, up to 10 years after implantation in humans (Hak, 2007). ProOsteon ${ }^{\circledR 500 R}$ is claimed to be more quickly degradable compared to ProOsteon 500 but complete degradation still requires at least 1 year in humans (Hak, 2007).

AEBVF implants showed near-complete degradation. AEBVF degradation rates are likely increased by incorporating morselised CBVF, exposing internal calcium carbonate that degrades much more quickly in vivo than the HA outer covering. Webster et al. reported effects of hydroxyapatite 
thickness influencing degradation rate in HAcoated implants, finding increased osteoclastic HA resorption with decreased grain size (Webster et al., 2001). Furthermore, HA-coated calcium carbonate exhibits much more rapid resorption than HA alone (McLaren et al., 2014). Faster AEBVF degradation rates may contribute to greater bone growth by increasing device porosity for bone infiltration (Hing et al., 2007).

This study has apparent limitations. The AEBVF evaluated here was only challenged against one $S$. aureus strain. Future work should evaluate additional strains and pathogens. Additionally, no AEBVF cohort without antibiotic was tested. Therefore, the effect of tobramycin release on the device's osteoconductivity cannot be determined. Finally, the goal of this study was to evaluate the device's ability to prevent infection. Future studies will test the device's ability to eradicate an established infection.

\section{Conclusion}

This large animal implant study validated this AEBVF as osteoconductive, in situ degradable, and able to eradicate $S$. aureus contamination at $5 \times 10^{5} \mathrm{CFU}$; thus, preventing infection. While all groups, except the ICo, exhibited bone formation, the AEBVF with or without $S$. aureus inoculum promoted new bone formation comparable to the commercial bone void filler (ProOsteon ${ }^{\circledR 500 R}$ ). Though these results warrant further testing, the AEBVF exhibits strong capabilities to prevent infection in contaminated bone defect procedures by eliminating clinically relevant bacterial challenges while facilitating new bone formation. In the future, an evaluation of the AEBVF technology is planned in a more clinically relevant two-stage surgical approach with confirmed osteomyelitis.

\section{Acknowledgements}

This work was supported by Elute, Inc. Dr. Grainger has equity in Elute, Inc. and Dr. Sinclair is a parttime employee. The authors acknowledge Drs. K. Andriano, B. Evans, and D. Evans for technical support; Mr. R. Olsen and the University of Utah's Office of Comparative Medicine for assistance with the large animal surgical study; and the University of Utah's Surface Analysis Laboratory for electron microscopy resources, and Elute, Inc. for financial support.

Zachary D. Ferrell conducted experiments and prepared the first draft of the manuscript; David W. Grainger helped with experimental design and critically revised the manuscript; Kristofer D. Sinclair designed and performed experiments, interpreted the data, and critically revised the manuscript. All authors have read and approved the final submitted manuscript.

\section{References}

An YH, Kang QK, Arciola CR (2006) Animal models of osteomyelitis. Int J Artif Organs 29: 407-420.

Bohm ER, Dunbar MJ, Frood JJ, Johnson TM, Morris KA (2012) Rehospitalizations, early revisions, infections, and hospital resource use in the first year after hip and knee arthroplasties. J Arthroplasty 27: 232-237.

Boyde A, Corsi A, Quarto R, Cancedda R, Bianco $P$ (1999) Osteoconduction in large macroporous hydroxyapatite ceramic implants: evidence for a complementary integration and disintegration mechanism. Bone 24: 579-589.

Brooks BD, Sinclair KD, Grainger DW, Brooks AE (2015) A resorbable antibiotic-eluting polymer composite bone void filler for perioperative infection prevention in a rabbit radial defect model. PLoS One 10: e0118696.

Campana V, Milano G, Pagano E, Barba M, Cicione C, Salonna G, Lattanzi W, Logroscino G (2014) Bone substitutes in orthopaedic surgery: from basic science to clinical practice. J Mater Sci Mater Med 25: 24452461.

Chai F, Hornez JC, Blanchemain N, Neut C, Descamps M, Hildebrand HF (2007) Antibacterial activation of hydroxyapatite (HA) with controlled porosity by different antibiotics. Biomol Eng 24: 510514.

Checketts RG, MacEachern AG, Otterburn M (2000) Pin Track infection and the principles of pin site care. Berlin/Heidleberg/New York: Springer; pp 97-103.

Clinical and Laboratory Standards Institute, (CLSI) (2006) M7-A7 Methods for dilution antimicrobial susceptibility tests for bacteria that grow aerobically. 26: 3-36.

El-Husseiny M, Patel S, MacFarlane RJ, Haddad FS (2011) Biodegradable antibiotic delivery systems. J Bone Joint Surg Br 93: 151-157.

Fletcher MD, Spencer RF, Langkamer VG, Lovering AM (2004) Gentamicin concentrations in diagnostic aspirates from 25 patients with hip and knee arthroplasties. Acta Orthop Scand 75: 173-176.

Goldman RT, Scuderi GR, Insall JN (1996) 2-stage reimplantation for infected total knee replacement. Clin Orthop Relat Res 331: 118-124.

Hak DJ. (2007) The use of osteoconductive bone graft substitutes in orthopaedic trauma. J Am Acad Orthop Surg 15: 525-536.

Harms A, Maisonneuve E, Gerdes K (2016) Mechanisms of bacterial persistence during stress and antibiotic exposure. Science 354: doi:10.1126/science. aaf4268

Hing KA, Wilson LF, Buckland T (2007) Comparative performance of three ceramic bone graft substitutes. Spine J 7: 475-490.

Jämsen E, Stogiannidis I, Malmivaara A, Pajamäki J, Puolakka T, Konttinen YT (2009) Outcome of prosthesis exchange for infected knee arthroplasty: 
the effect of treatment approach. Acta Orthop 80: 67-77.

Jones Z, Brooks AE, Ferrell Z, Grainger DW, Sinclair KD (2016) A resorbable antibiotic eluting bone void filler for periprosthetic joint infection prevention. J Biomed Mater Res B Appl Biomater 104: 1632-1642.

Kurtz SM, Lau E, Watson H, Schmier JK, Parvizi J (2012) Economic burden of periprosthetic joint infection in the United States. J Arthroplasty 27: 61-65.

McLaren JS, White LJ, Cox HC, Ashraf W, Rahman CV, Blunn GW, Goodship AE, Quirk RA, Shakesheff KM, Bayston R, Scammell BE (2014) A biodegradable antibiotic-impregnated scaffold to prevent osteomyelitis in a contaminated in vivo bone defect model. Eur Cell Mater 27: 332-349.

Miyai T, Ito A, Tamazawa G, Matsuno T, Sogo Y, Nakamura C, Yamazaki A, Satoh T (2008) Antibioticloaded poly-epsilon-caprolactone and porous beta-tricalcium phosphate composite for treating osteomyelitis. Biomaterials 29: 350-358.

Rao N, Cannella B, Crossett LS, Yates AJ, McGough $\mathrm{R}$ (2008) A preoperative decolonization protocol for Staphylococcus aureus prevents orthopaedic infections. Clin Orthop Relat Res 466: 1343-1348.

Reddi AH (1985) Implant-stimulated interface reactions during collagenous bone matrix-induced bone formation. J Biomed Mater Res 19: 233-239.

Reizner W, Hunter JG, O'Malley NT, Southgate RD, Schwarz EM, Kates SL (2014) A systematic review of animal models for Staphylococcus aureus osteomyelitis. Eur Cell Mater 27: 196-212.

Sinclair KD, Pham TX, William DL, Farnsworth R, Loc-Carrillo C, Bloebaum R (2013) Model development for determining the efficacy of a combination coating for the prevention of perioperative device related infections: a pilot study. J Biomed Mater Res B Appl Biomater 101: 1143-1153.

Sørensen TS, Sørensen AI, Merser S (1990) Rapid release of gentamicin from collagen sponge. In vitro comparison with plastic beads. Acta Orthop Scand 61: 353-356.

van de Belt $H$, Neut D, Schenk W, van Horn JR, van der Mei HC, Busscher HJ (2000) Gentamicin release from polymethylmethacrylate bone cements and Staphylococcus aureus biofilm formation. Acta Orthop Scand 71: 625-629.

Von Stechow D, Scale D, Rauschmann MA (2005) Minimizing the surgical approach in patients with spondylitis. Clin Orthop Relat Res. 439: 61-67.

Webster TJ, Ergun C, Doremus RH, Siegel RW, Bizios R (2001) Enhanced osteoclast-like cell functions on nanophase ceramics. Biomaterials 22: 1327-1333.

\section{Web References}

1. Pfunner A, Wier L, Stocks C. (2013). Most frequent procedures performed in U.S. hospitals, 2010. HCUP Statistical Brief \#149. Available: http://www.hcupus. ahrq.gov/reports/statbriefs/sb149.pdf. [12-11-2016].

\section{Discussion with Reviewer}

Volker Alt: The use of resorbable polymers in orthopaedics is questionable because their biocompatibility is related to the implanted volume. Can the authors speculate on the maximum amount of AEBVF that can be implanted without causing biocompatibility problems?

Authors: Previous work has shown that these devices cannot be used with the traditional "line-to-line" "fit and fill" approach. Fluid flow around the devices is required; thus, the loose device packing noted by the reviewer. This speaks to the requirement to provide passive biotransport, fluid fluxes, cellular infiltrates and metabollte exchange around and eventually throughout the implant. This determines the resoprtion rate and maximum implant volume. That said, clinically relevant voids (up to $25 \mathrm{~mm}$ diameter) associated with TJR should be filled with new bone in 8-12 weeks.

Editor's note: The Scientific Editor responsible for this paper was Fintan Moriaty. 